\title{
Ethnic inequalities in hospital admissions in England: an observational study
}

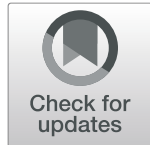

Jakob Petersen ${ }^{1 *}$, Jens Kandt ${ }^{2}$ and Paul A. Longley ${ }^{1}$

\begin{abstract}
Background: Ethnic inequalities in health are well-known and partly explained by social determinants such as poorer living and working conditions, health behaviours, discrimination, social exclusion, and healthcare accessibility factors. Inequalities are known both for self-reported health and for diseases such as diabetes, cardiovascular diseases, respiratory diseases, and non-specific chest pains. Most studies however concern individual diseases or self-reported health and do not provide an overview that can detect gaps in existing knowledge. The aim of this study is thus to identify ethnic inequalities in inpatient hospital admission for all major disease categories in England.

Methods: Observational study of the inpatient hospital admission database in England enhanced with ethnicity coding of participants' surnames. The primary diagnosis was coded to Level 1 of the Global Burden of Disease groups. For each year, only the first admission for each condition for each participant was included. If a participant was readmitted within two days only the first admission was counted. Admission risk for all major disease groups for each ethnic group relative to the White British group were calculated using logistic regression adjusting for age and area deprivation.

Results: 40,928,105 admissions were identified between April 2009 and March 2014. Ethnic inequalities were found in cardiovascular diseases, respiratory diseases, chest pain, and diabetes in line with previous studies. Additional inequalities were found in nutritional deficiencies, endocrine disorders, and sense organ diseases.

Conclusions: The results of this study were consistent with known inequalities, but also found previously unreported disparities in nutritional deficiencies, endocrine disorders, and sense organ diseases. Further studies would be required to map out the relevant care pathways for ethnic minorities and establish whether preventive measures can be strengthened.
\end{abstract}

Keywords: Health disparities, Ethnicity, Departments, hospital, Electronic health records

\section{Introduction}

Reducing inequalities in health has explicitly been part of the government agenda in the United Kingdom (UK) since 1997 [1, 2]. Ethnic inequalities in health are mainly caused by poorer living and working conditions, discrimination, marginalisation and differences in health and health seeking behaviours [3-7]. In exceptional cases,

\footnotetext{
* Correspondence: jakob.petersen1@|shtm.ac.uk

${ }^{1}$ Consumer Data Research Centre (CDRC), Department of Geography, University College London (UCL), Gower Street, London WC1E 6BT, UK

Full list of author information is available at the end of the article
}

some groups are genetically predisposed to certain diseases such as sickle cell anaemia [3, 7]. Major conditions with known ethnic inequalities include diabetes, respiratory diseases, cardiovascular diseases, and non-specific chest pains [3-7]. National Health Service (NHS) hospitals monitor their use by ethnic group in the national database, Hospital Episode Statistics (HES), so that inequalities can be detected and ameliorated through adjustments in care provision and preventive action [8]. In this study we analyse hospital admission records by ethnic group across all major disease categories in the

(c) The Author(s). 2021 Open Access This article is licensed under a Creative Commons Attribution 4.0 International License, which permits use, sharing, adaptation, distribution and reproduction in any medium or format, as long as you give appropriate credit to the original author(s) and the source, provide a link to the Creative Commons licence, and indicate if changes were made. The images or other third party material in this article are included in the article's Creative Commons licence, unless indicated otherwise in a credit line to the material. If material is not included in the article's Creative Commons licence and your intended use is not permitted by statutory regulation or exceeds the permitted use, you will need to obtain permission directly from the copyright holder. To view a copy of this licence, visit http://creativecommons.org/licenses/by/4.0/ The Creative Commons Public Domain Dedication waiver (http://creativecommons.org/publicdomain/zero/1.0/) applies to the data made available in this article, unless otherwise stated in a credit line to the data. 
Global Burden of Disease (GBD) classification in 20092013 [9]. The study is intended to identify health inequalities across a wide spectrum of health conditions among in-patient admissions across England and its regions. There is an extensive literature on ethnic inequalities in health in the UK [3], but usually these pertain to individual diseases or self-reported health and do not provide an overview that can detect gaps in existing knowledge.

\section{Methods}

Hospital admission records were obtained from NHS England's Hospital Episode Statistics (HES), April 2009March 2014 (financial years 2009-2013). Because some ethnic groups are relatively small, data on hospital admission for five years were analysed assuming that the 2011 Census population would be representative of the at-risk populations that year as well as two years before and two years after. Diagnoses in HES are coded to the 10th version of International Classification of Diseases (ICD10) system [10]. Data on the primary diagnosis for each admission were for the purpose of this study coded with definitions for the GBD conditions (Level 1). This high-level disease classification was developed for international comparison of health and healthcare factors, e.g. Cardiovascular diseases (2G) or Respiratory diseases $(2 \mathrm{H})$ [9]. If a patient was re-admitted within two days, only the first admission was counted. If a patient had more than one admission for the same condition in a year, only the first admission was kept. Area deprivation quintiles were coded at local authority level [11].

There is a growing literature about the use of names to impute ethnicity in studies where this information is not routinely collected or not otherwise available through data linkage [6, 12-14]. Software developed at University College London [13, 15] has been used in over 60 scientific studies and social equity audits in applications as diverse as accident and emergency department utilisation [16], residential segregation [17], labour market discrimination [18], and the composition of company boards [19]. To address problems with missing self-reported ethnicity information in HES, the Ethnicity Estimator (EE) software was deployed in this study [12, 13]. To retain full anonymity, this step was carried out in an air-gapped, secure data facility by NHS Digital linking name information in the Patient Demographic Service to HES. The sensitivity of the EE software in predicting the self-reported ethnic group was calculated. This study was part of a bigger project that reconciled ethnicity data within NHS over time (1999-2014) and between NHS, Censuses 2001 and 2011, and aggregation of ethnic groups in census outputs for small areas. "Arab" was grouped with "Other" in census outputs as there is no category for "Arab" in the NHS ethnicity classification. "Black other" was grouped with "Other" to reconcile how coded and aggregated in Census data tables for small areas. Preliminary work showed that surname imputation tends to inflate the White Irish group relative to self-reported data and the admission results for White Irish with imputed data are not shown.

The odds ratios of admission for different ethnic minorities were estimated with White British group as reference using logistic regression controlling for age in 20 -year bands between 0 and 99 years of age and area deprivation quintile for each condition and all-cause hospitalisation. The analyses were repeated without surname imputation for sensitivity.

To identify geographic inequalities, the incidence of all-cause hospitalisation was calculated at local authority level and standardised by age and sex using the Census 2011 denominators and 2013 European Standard Population weights [20]. Counts below 20 cases were suppressed. Ethical approval was obtained from Bromley REC (Reference: 13/LO/1355) for analyses of patientlevel HES data. The HES data licence reference is DARS-NIC-28051-Q3K7L.

\section{Results}

There was a total of 40,928,105 admissions of 24,997,325 patients in 2009/13 of which $9.7 \%$ had missing selfreported ethnicity (Table 1). The total number of admissions by condition can be found in Table 2 .

The regression analyses showed a diverse picture where ethnic minorities have significantly higher admission for some conditions and lower for others (Table 3). These include higher risks for e.g. cardiovascular diseases for Bangladeshi and Pakistani or higher diabetes admission risk for the Black Caribbean group. It also showed that Pakistani, Other, and White other had higher admission risk than the White British group for most conditions, while Chinese and mixed ethnicity had significantly lower admission risk. Nutritional deficiencies were particular high for Bangladeshi, Pakistani, and Other (the dominant nutritional deficiency for all ethnic groups was iron deficiency anaemia). Endocrine disorders were higher in Black African, Black Caribbean, and Other (the dominant endocrine disorder for these groups were sickle-cell disorders). Sense organ diseases were higher in Other and South Asian groups (the dominant sense organ condition for all ethnic groups was cataracts). Non-specific chest pain admission, an early symptom of cardiovascular disease, was notably also very high in South Asians and the Other group. The risks deattenuated in analyses adjusted for area deprivation quintile in addition to age, especially for high-risk groups, which indicates that higher admission risk was partly explained by residence in deprived regions (Table 4). 
Table 1 NHS-recorded and EE-imputed ethnicity on HES admissions, 2009-2013

\begin{tabular}{|c|c|c|c|c|}
\hline Ethnic Group & NHS-recorded & $\%$ & NHS-recorded with EE imputed & $\%$ \\
\hline Asian Other & 420,374 & 1.0 & 466,531 & 1.1 \\
\hline Bangladeshi & 224,393 & 0.6 & 251,056 & 0.6 \\
\hline Chinese & 117,965 & 0.3 & 140,002 & 0.3 \\
\hline Indian & 748,626 & 1.8 & 862,019 & 2.1 \\
\hline Pakistani & 742,705 & 1.8 & 856,327 & 2.1 \\
\hline Black African & 498,148 & 1.2 & 572,557 & 1.4 \\
\hline Black Caribbean & 377,545 & 0.9 & 395,783 & 1.0 \\
\hline Other & 907,138 & 2.2 & 939,075 & 2.3 \\
\hline White Other & $1,623,917$ & 4.0 & $1,903,032$ & 4.7 \\
\hline White British & $30,610,616$ & 74.8 & $33,540,602$ & 82.0 \\
\hline White Irish & 273,845 & 0.7 & 466,241 & 1.1 \\
\hline Mixed & 409,095 & 1.0 & 409,095 & 1.0 \\
\hline Missing & $3,973,738$ & 9.7 & 125,785 & 0.3 \\
\hline Total & $40,928,105$ & 100 & $40,928,105$ & 100 \\
\hline
\end{tabular}

For all-cause hospitalisation the risk was particularly high among patients with Pakistani (OR 1.28; 95\% CI $1.28-1.29)$ and Other $(1.83 ; 1.83-1.83)$ background compared to White British (Table 5; Table S3 for analysis without surname imputation). The maps showed that the risk of hospitalisation for White British was generally higher in Northern metropolitan areas (Fig. 1). For Pakistani (Fig. 2) and Other (Fig. 3), ethnic groups with higher risk of admission compared to White British, the regional distribution was more complex and widespread.

When the records with missing ethnicity was replaced with EE categories, only $0.3 \%$ of admissions where without ethnicity coding. The sensitivity of the EE-prediction relative to NHS-recorded ethnicity varied by ethnic group: White British (89.6\%), Pakistani (80.7\%), Indian (73.9\%), Chinese (71.0\%), Bangladeshi (63.0\%), Black African (54.9\%), White Other (46.2\%), White Irish (44.8\%), Asian Other (20.7\%), Black Caribbean (9.5\%), Other (4.9\%), and Mixed (0\%). The regression analyses were repeated without the surname imputation for sensitivity and yielded similar results (Table S1, S2, S3, Supplementary materials).

\section{Discussion}

Ethnic inequalities in health are well-known and partly explained by a combination of poorer living and working conditions and differences in health and health seeking behaviours [3-7]. The results of this study were consistent with known inequalities for diabetes, cardiovascular diseases, respiratory diseases, non-specific chest pains as well as previously unreported disparities in nutritional deficiencies, endocrine disorders, and sense organ diseases.

South Asians, Pakistani and Bangladeshi groups more than Indian, have relatively high risk of diabetes, coronary heart disease [21-24], asthma [5, 25], and certain gastrointestinal diseases [26]. The Pakistani group is also associated with higher risk of respiratory disease admission [27].

Black ethnic groups have higher risk of hypertension and diabetes [21]. Older studies showed higher risk of asthma admission among Black ethnic groups [28], while a more recent study did not find a higher risk of first asthma admission in Black ethnic groups compared to the White British group [25].

The results of this study confirmed previously reported risks for cardiovascular diseases, chest pain, and respiratory diseases with a few exceptions, e.g. Bangladeshis were not strongly associated with diabetes and respiratory disease admission and South Asians were not associated with gastrointestinal diseases. The reason for the latter is likely to be that the previous study only found an association for two less common gastrointestinal diseases, ulcerative colitis and Crohn's disease [26], and that any association could have been swamped out by more common gastrointestinal disorders less specific to any particular ethnic group.

For diabetes, the Black Caribbean group had more than twice the admission risk of the White British group. The results also showed inequalities in areas that - to our knowledge - have not previously been reported in the UK, i.e. higher risk of nutritional deficiencies (Bangladeshi and Pakistani), endocrine disorders (Black 
Table 2 Total number of admissions by Global Burden of Disease (Level 1) category in England, 2009-2013

\begin{tabular}{|c|c|c|}
\hline GBD Level 1 Conditions & Freq. & $\%$ \\
\hline 1A Infectious and parasitic diseases & 828,652 & 2.0 \\
\hline 1B Respiratory infections & $1,458,212$ & 3.6 \\
\hline 1C Maternal conditions & $4,469,195$ & 10.9 \\
\hline 1D Perinatal conditions & 455,236 & 1.1 \\
\hline 1E Nutritional deficiencies & 330,211 & 0.8 \\
\hline 2A Malignant neoplasms & $1,566,246$ & 3.8 \\
\hline 2B Other neoplasms & 981,990 & 2.4 \\
\hline 2C Diabetes mellitus & 160,722 & 0.4 \\
\hline 2D Endocrine disorders & 515,230 & 1.3 \\
\hline 2E Neuro-psychiatric conditions & $1,965,073$ & 4.8 \\
\hline 2F Sense organ diseases & $2,078,647$ & 5.1 \\
\hline 2G Cardiovascular diseases & $2,699,978$ & 6.6 \\
\hline 2H Respiratory diseases & $1,485,766$ & 3.6 \\
\hline 2l Digestive diseases & $4,994,943$ & 12.2 \\
\hline $2 \mathrm{~J}$ Genito-urinary diseases & $3,166,208$ & 7.7 \\
\hline 2 K Skin diseases & $1,124,447$ & 2.7 \\
\hline $2 \mathrm{~L}$ Musculoskeletal diseases & $3,981,799$ & 9.7 \\
\hline $2 \mathrm{M}$ Congenital anomalies & 307,166 & 0.8 \\
\hline $2 \mathrm{~N}$ Oral conditions & $1,187,828$ & 2.9 \\
\hline 30 Injuries & $3,140,995$ & 7.7 \\
\hline X102 Nonspecific chest pain & 882,626 & 2.2 \\
\hline X176 Contraceptive and procreative management & 150,778 & 0.4 \\
\hline X251 Abdominal pain & 945,531 & 2.3 \\
\hline X257 Other aftercare & 551,236 & 1.3 \\
\hline X259 Residual codes - unclassified & 402,126 & 1.0 \\
\hline XR Symptoms signs and abnormal clinical and laboratory findings & 528,269 & 1.3 \\
\hline XZ Factors influencing health status and contact with health services & 568,870 & 1.4 \\
\hline Other & 126 & 0.0 \\
\hline Total & $40,928,105$ & 100 \\
\hline
\end{tabular}

African, Black Caribbean, and Other), and sense organ diseases (South Asian and Other).

Nutritional deficiencies can generally be classed as preventable and further studies would be required to identify relevant preventive measures for the identified ethnic groups, especially Bangladeshi, Pakistani and Other. The dominant nutritional deficiency was iron deficiency anaemia, which can be associated with dietary deficiency, increased loss or requirement, or malabsorption due to underlying health conditions [29].

This high-level analysis revealed ethnic inequalities in endocrine disorders for the Black African group, Black Caribbean group, and the Other group. The dominant endocrine disorder for these groups were sickle-cell disorders, which are rare genetic disorders typically found in persons with ancestors from sub-Saharan Africa [30].

Sense organ diseases were higher in Other and South Asian groups. The dominant sense organ condition for all ethnic groups was cataracts. This pattern is consistent with a UK survey of South Asian patients that showed higher cataract prevalence than the general population of the UK and the US [31].

The overall results show that ethnic disparities are wide ranging and involving many different care pathways. For some of these pathways there are well established secondary preventive care in the primary and community care sector, e.g. the monitory of patients with diabetes or heart conditions in general 
Table 3 Age-adjusted odds ratios of admission incidence relative to White British group. Blue shading shows odds ratios significantly below White British group; red shading above. Darker tones show less than half and more than twice, respectively. Nonsignificant (ns) results shown on background. NHS-recorded ethnicity replaced with EE prediction where missing

\begin{tabular}{|c|c|c|c|c|c|c|c|c|c|c|c|}
\hline $\begin{array}{l}\text { GB } \\
\text { D1a }\end{array}$ & Description & $\begin{array}{l}\text { Asian } \\
\text { other }\end{array}$ & $\begin{array}{c}\text { Bangla } \\
\text { deshi }\end{array}$ & Chinese & Indian & $\begin{array}{c}\text { Pakista } \\
\text { ni }\end{array}$ & $\begin{array}{c}\text { Black } \\
\text { African }\end{array}$ & $\begin{array}{c}\text { Black } \\
\text { Caribbe } \\
\text { an }\end{array}$ & Other & $\begin{array}{l}\text { White } \\
\text { Other }\end{array}$ & Mixed \\
\hline $1 \mathrm{~A}$ & $\begin{array}{l}\text { Infectious and } \\
\text { parasitic } \\
\text { diseases }\end{array}$ & 1.12 & $1.00 \mathrm{~ns}$ & 0.79 & 1.12 & 1.64 & 1.14 & 0.89 & 1.79 & 1.16 & 0.67 \\
\hline 1B & $\begin{array}{l}\text { Respiratory } \\
\text { infections }\end{array}$ & 0.88 & 0.93 & 0.47 & 0.89 & 1.45 & 0.78 & 0.70 & 1.47 & 1.15 & 0.55 \\
\hline $1 \mathrm{C}$ & $\begin{array}{l}\text { Maternal } \\
\text { conditions }\end{array}$ & 1.01 & 1.22 & 0.65 & 0.96 & 1.56 & 1.47 & 1.03 & 1.87 & 1.28 & 0.70 \\
\hline 1D & $\begin{array}{l}\text { Perinatal } \\
\text { conditions }\end{array}$ & 1.32 & 1.16 & 1.20 & 1.54 & 1.39 & 1.29 & 1.03 & 1.65 & 2.80 & 0.94 \\
\hline $1 \mathrm{E}$ & $\begin{array}{l}\text { Nutritional } \\
\text { deficiencies }\end{array}$ & 1.32 & 2.33 & 0.70 & 1.86 & 2.58 & 1.43 & 1.27 & 2.16 & 1.15 & 0.69 \\
\hline $2 \mathrm{~A}$ & $\begin{array}{l}\text { Malignant } \\
\text { neoplasms }\end{array}$ & 0.53 & 0.52 & 0.54 & 0.49 & 0.59 & 0.67 & 0.71 & 1.35 & 1.23 & 0.44 \\
\hline $2 \mathrm{~B}$ & $\begin{array}{l}\text { Other } \\
\text { neoplasms }\end{array}$ & 0.72 & 0.68 & 0.70 & 0.71 & 0.81 & 1.21 & 1.24 & 1.65 & 1.16 & 0.58 \\
\hline $2 \mathrm{C}$ & $\begin{array}{l}\text { Diabetes } \\
\text { mellitus }\end{array}$ & 0.89 & 0.93 & 0.39 & 1.13 & 1.51 & 1.30 & 2.31 & 2.14 & 0.92 & 0.63 \\
\hline $2 \mathrm{D}$ & $\begin{array}{l}\text { Endocrine } \\
\text { disorders }\end{array}$ & 0.98 & 1.07 & 0.65 & 1.09 & 1.67 & 1.87 & 1.84 & 2.22 & 1.12 & 0.64 \\
\hline $2 \mathrm{E}$ & $\begin{array}{l}\text { Neuro- } \\
\text { psychiatric } \\
\text { conditions }\end{array}$ & 0.80 & 0.78 & 0.33 & 0.77 & 1.03 & 0.93 & 1.16 & 1.88 & 1.05 & 0.62 \\
\hline $2 \mathrm{~F}$ & $\begin{array}{l}\text { Sense organ } \\
\text { diseases }\end{array}$ & 1.32 & 1.58 & 0.93 & 1.62 & 1.98 & 1.46 & 1.26 & 2.16 & 1.39 & 0.64 \\
\hline $2 \mathrm{G}$ & $\begin{array}{l}\text { Cardiovascula } \\
\mathrm{r} \text { diseases }\end{array}$ & 1.07 & 1.35 & 0.63 & 1.15 & 1.65 & 0.89 & 0.93 & 2.01 & 1.37 & 0.57 \\
\hline $2 \mathrm{H}$ & $\begin{array}{l}\text { Respiratory } \\
\text { diseases }\end{array}$ & 1.03 & 1.18 & 0.49 & 1.02 & 1.71 & 0.93 & 0.90 & 1.83 & 1.12 & 0.65 \\
\hline $2 \mathrm{I}$ & $\begin{array}{l}\text { Digestive } \\
\text { diseases }\end{array}$ & 0.81 & 1.02 & 0.54 & 0.88 & 1.15 & 0.74 & 0.80 & 1.56 & 1.08 & 0.50 \\
\hline $2 \mathrm{~J}$ & $\begin{array}{l}\text { Genito- } \\
\text { urinary } \\
\text { diseases }\end{array}$ & 0.85 & 0.91 & 0.53 & 0.91 & 1.14 & 0.83 & 1.01 & 1.74 & 1.14 & 0.55 \\
\hline $2 \mathrm{~K}$ & Skin diseases & 0.83 & 0.77 & 0.46 & 0.89 & 1.26 & 0.76 & 0.88 & 1.60 & 1.03 & 0.52 \\
\hline $2 \mathrm{~L}$ & $\begin{array}{l}\text { Musculoskelet } \\
\text { al diseases }\end{array}$ & 0.58 & 0.52 & 0.25 & 0.74 & 0.83 & 0.59 & 0.75 & 1.25 & 0.92 & 0.47 \\
\hline $2 \mathrm{M}$ & $\begin{array}{l}\text { Congenital } \\
\text { anomalies }\end{array}$ & 0.90 & 0.77 & 0.63 & 0.85 & 1.24 & 0.92 & 0.79 & 1.31 & 1.32 & 0.60 \\
\hline $2 \mathrm{~N}$ & $\begin{array}{l}\text { Oral } \\
\text { conditions }\end{array}$ & 0.78 & 0.84 & 0.55 & 0.72 & 1.04 & 0.78 & 1.31 & 1.62 & 0.92 & 0.57 \\
\hline 30 & Injuries & 0.64 & 0.47 & 0.33 & 0.56 & 0.75 & 0.53 & 0.66 & 1.57 & 0.96 & 0.46 \\
\hline $\begin{array}{l}\mathrm{X} 10 \\
2 \\
\end{array}$ & $\begin{array}{l}\text { Non specific } \\
\text { chest pain }\end{array}$ & 1.40 & 2.10 & 0.41 & 1.45 & 3.00 & 1.11 & 1.16 & 2.47 & 1.23 & 0.63 \\
\hline $\begin{array}{l}\text { X17 } \\
6\end{array}$ & $\begin{array}{l}\text { Contraceptive } \\
\text { and } \\
\text { procreative } \\
\text { management }\end{array}$ & 0.49 & 0.58 & 0.33 & 0.54 & 0.69 & 0.84 & 0.82 & 1.09 & 0.63 & 0.46 \\
\hline $\begin{array}{l}\mathrm{X} 25 \\
1\end{array}$ & $\begin{array}{l}\text { Abdominal } \\
\text { pain }\end{array}$ & 0.79 & 0.83 & 0.43 & 0.79 & 1.29 & 0.79 & 0.86 & 1.62 & 0.88 & 0.47 \\
\hline $\begin{array}{l}\mathrm{X} 25 \\
7 \\
\end{array}$ & $\begin{array}{l}\text { Other } \\
\text { aftercare }\end{array}$ & 0.60 & 0.51 & 0.51 & 0.57 & 0.70 & 0.57 & 0.66 & 1.25 & 1.14 & 0.46 \\
\hline $\begin{array}{l}\text { X25 } \\
9\end{array}$ & $\begin{array}{l}\text { Residual } \\
\text { codes } \\
\text { unclassified }\end{array}$ & 0.89 & 1.46 & 0.55 & 0.79 & 1.16 & 1.12 & 1.25 & 1.89 & 1.27 & 0.74 \\
\hline $\mathrm{XR}$ & $\begin{array}{l}\text { Symptoms, } \\
\text { signs and } \\
\text { abnormal } \\
\text { clinical and } \\
\text { laboratory } \\
\text { findings }\end{array}$ & 1.14 & 1.11 & 0.59 & 1.08 & 1.54 & 1.10 & 1.04 & 2.09 & 1.19 & 0.63 \\
\hline $\mathrm{XZ}$ & $\begin{array}{l}\text { Factors } \\
\text { influencing } \\
\text { health status } \\
\text { and contact } \\
\text { with health } \\
\text { services }\end{array}$ & 0.95 & $1.00 \mathrm{~ns}$ & 0.77 & 1.07 & 1.35 & 1.17 & 1.05 & 1.79 & 1.37 & 0.69 \\
\hline
\end{tabular}


Table 4 Age- and deprivation-adjusted odds ratios of admission incidence relative to White British group. Blue shading shows odds ratios significantly below White British group; red shading above. Darker tones show less than half and more than twice, respectively. Non-significant (ns) results shown on background. NHS-recorded ethnicity replaced with EE prediction where missing

\begin{tabular}{|c|c|c|c|c|c|c|c|c|c|c|c|}
\hline $\begin{array}{l}\text { GB } \\
\text { D1a }\end{array}$ & Description & $\begin{array}{l}\text { Asian } \\
\text { other }\end{array}$ & $\begin{array}{c}\text { Bangla } \\
\text { deshi }\end{array}$ & Chinese & Indian & $\begin{array}{c}\text { Pakista } \\
\text { ni }\end{array}$ & $\begin{array}{c}\text { Black } \\
\text { African }\end{array}$ & $\begin{array}{c}\text { Black } \\
\text { Caribbe } \\
\text { an }\end{array}$ & Other & $\begin{array}{l}\text { White } \\
\text { Other }\end{array}$ & Mixed \\
\hline $1 \mathrm{~A}$ & $\begin{array}{l}\text { Infectious and } \\
\text { parasitic } \\
\text { diseases }\end{array}$ & 1.08 & 0.91 & 0.76 & 1.07 & 1.49 & 1.05 & 0.81 & 1.66 & 1.13 & 0.65 \\
\hline 1B & $\begin{array}{l}\text { Respiratory } \\
\text { infections }\end{array}$ & 0.86 & 0.83 & 0.46 & 0.85 & 1.32 & 0.72 & 0.63 & 1.36 & 1.12 & 0.53 \\
\hline $1 \mathrm{C}$ & $\begin{array}{l}\text { Maternal } \\
\text { conditions }\end{array}$ & $1.00 \mathrm{~ns}$ & 1.21 & 0.65 & 0.95 & 1.55 & 1.46 & 1.02 & 1.86 & 1.28 & 0.70 \\
\hline $1 \mathrm{D}$ & $\begin{array}{l}\text { Perinatal } \\
\text { conditions }\end{array}$ & 1.31 & 1.13 & 1.19 & 1.52 & 1.35 & 1.26 & $1.00 \mathrm{~ns}$ & 1.61 & 2.78 & 0.93 \\
\hline $1 \mathrm{E}$ & $\begin{array}{l}\begin{array}{l}\text { Nutritional } \\
\text { deficiencies }\end{array} \\
\end{array}$ & 1.29 & 2.18 & 0.68 & 1.79 & 2.42 & 1.34 & 1.20 & 2.06 & 1.14 & 0.67 \\
\hline $2 \mathrm{~A}$ & $\begin{array}{l}\text { Malignant } \\
\text { neoplasms }\end{array}$ & 0.53 & 0.51 & 0.54 & 0.49 & 0.58 & 0.66 & 0.69 & 1.33 & 1.22 & 0.43 \\
\hline $2 \mathrm{~B}$ & $\begin{array}{l}\text { Other } \\
\text { neoplasms }\end{array}$ & 0.71 & 0.66 & 0.69 & 0.69 & 0.79 & 1.18 & 1.20 & 1.62 & 1.15 & 0.57 \\
\hline $2 \mathrm{C}$ & $\begin{array}{l}\text { Diabetes } \\
\text { mellitus }\end{array}$ & 0.86 & 0.82 & 0.38 & 1.07 & 1.35 & 1.19 & 2.08 & 2.08 & 0.89 & 0.60 \\
\hline $2 \mathrm{D}$ & $\begin{array}{l}\text { Endocrine } \\
\text { disorders }\end{array}$ & 0.95 & 0.97 & 0.63 & 1.04 & 1.53 & 1.74 & 1.70 & 2.08 & 1.10 & 0.62 \\
\hline $2 \mathrm{E}$ & $\begin{array}{l}\text { Neuro- } \\
\text { psychiatric } \\
\text { conditions }\end{array}$ & 0.78 & 0.71 & 0.32 & 0.74 & 0.95 & 0.87 & 1.07 & 1.78 & 1.03 & 0.60 \\
\hline $2 \mathrm{~F}$ & $\begin{array}{l}\text { Sense organ } \\
\text { diseases }\end{array}$ & 1.30 & 1.50 & 0.91 & 1.58 & 1.89 & 1.40 & 1.21 & 2.09 & 1.38 & 0.63 \\
\hline $2 \mathrm{G}$ & $\begin{array}{l}\text { Cardiovascula } \\
\mathrm{r} \text { diseases }\end{array}$ & 1.05 & 1.26 & 0.62 & 1.12 & 1.56 & 0.85 & 0.88 & 1.99 & 1.36 & 0.55 \\
\hline $2 \mathrm{H}$ & $\begin{array}{l}\text { Respiratory } \\
\text { diseases }\end{array}$ & $1.00 \mathrm{~ns}$ & 1.04 & 0.47 & 0.96 & 1.53 & 0.85 & 0.81 & 1.70 & 1.09 & 0.62 \\
\hline $2 \mathrm{I}$ & $\begin{array}{l}\text { Digestive } \\
\text { diseases }\end{array}$ & 0.80 & 0.96 & 0.53 & 0.86 & 1.09 & 0.71 & 0.76 & 1.51 & 1.07 & 0.49 \\
\hline $2 \mathrm{~J}$ & $\begin{array}{l}\text { Genito- } \\
\text { urinary } \\
\text { diseases }\end{array}$ & 0.84 & 0.87 & 0.52 & 0.89 & 1.10 & 0.81 & 0.98 & 1.69 & 1.13 & 0.54 \\
\hline $2 \mathrm{~K}$ & Skin diseases & 0.81 & 0.71 & 0.45 & 0.85 & 1.17 & 0.71 & 0.82 & 1.51 & 1.01 & 0.51 \\
\hline $2 \mathrm{~L}$ & $\begin{array}{l}\text { Musculoskelet } \\
\text { al diseases }\end{array}$ & 0.58 & 0.51 & 0.25 & 0.73 & 0.82 & 0.58 & 0.74 & 1.22 & 0.92 & 0.47 \\
\hline $2 \mathrm{M}$ & $\begin{array}{l}\text { Congenital } \\
\text { anomalies }\end{array}$ & 0.89 & 0.73 & 0.62 & 0.84 & 1.20 & 0.90 & 0.76 & 1.27 & 1.30 & 0.59 \\
\hline $2 \mathrm{~N}$ & $\begin{array}{l}\text { Oral } \\
\text { conditions }\end{array}$ & 0.75 & 0.75 & 0.53 & 0.69 & 0.94 & 0.72 & 1.21 & 1.52 & 0.90 & 0.55 \\
\hline 30 & Injuries & 0.62 & 0.44 & 0.32 & 0.54 & 0.70 & 0.50 & 0.62 & 1.49 & 0.94 & 0.44 \\
\hline $\begin{array}{l}\text { X10 } \\
2\end{array}$ & $\begin{array}{l}\text { Non specific } \\
\text { chest pain }\end{array}$ & 1.37 & 1.89 & 0.39 & 1.38 & 2.73 & 1.02 & 1.06 & 2.31 & 1.20 & 0.61 \\
\hline $\begin{array}{l}\mathrm{X} 17 \\
6\end{array}$ & $\begin{array}{l}\text { Contraceptive } \\
\text { and } \\
\text { procreative } \\
\text { management }\end{array}$ & 0.47 & 0.53 & 0.31 & 0.51 & 0.64 & 0.78 & 0.75 & 1.02 & 0.62 & 0.44 \\
\hline $\begin{array}{l}\mathrm{X} 25 \\
1 \\
\end{array}$ & $\begin{array}{l}\text { Abdominal } \\
\text { pain }\end{array}$ & 0.77 & 0.78 & 0.41 & 0.77 & 1.21 & 0.75 & 0.81 & 1.54 & 0.86 & 0.45 \\
\hline $\begin{array}{l}\text { X25 } \\
7\end{array}$ & $\begin{array}{l}\text { Other } \\
\text { aftercare }\end{array}$ & 0.60 & 0.50 & 0.50 & 0.57 & 0.68 & 0.56 & 0.65 & 1.22 & 1.13 & 0.46 \\
\hline $\begin{array}{l}\mathrm{X} 25 \\
9\end{array}$ & $\begin{array}{l}\text { Residual } \\
\text { codes } \\
\text { unclassified }\end{array}$ & 0.88 & 1.37 & 0.54 & 0.78 & 1.10 & 1.08 & 1.19 & 1.83 & 1.25 & 0.73 \\
\hline $\mathrm{XR}$ & $\begin{array}{l}\text { Symptoms, } \\
\text { signs and } \\
\text { abnormal } \\
\text { clinical and } \\
\text { laboratory } \\
\text { findings } \\
\end{array}$ & 1.11 & $0.99 \mathrm{~ns}$ & 0.57 & 1.04 & 1.40 & 1.02 & 0.95 & 1.95 & 1.17 & 0.61 \\
\hline $\mathrm{XZ}$ & $\begin{array}{l}\text { Factors } \\
\text { influencing } \\
\text { health status } \\
\text { and contact } \\
\text { with health } \\
\text { services }\end{array}$ & 0.94 & 0.97 & 0.76 & 1.06 & 1.31 & 1.14 & 1.03 & 1.76 & 1.36 & 0.68 \\
\hline
\end{tabular}


Table 5 Age- and deprivation- adjusted odds ratios (OR 95\% Cl) for all-cause hospitalisation. NHS-recorded ethnicity replaced with EE prediction where missing

\begin{tabular}{lllllll}
\hline Ethnic group & Case $\mathbf{( N )}$ & No case $(\mathbf{N})$ & Age- adj. OR $\mathbf{( 9 5 \% ~ C l )}$ & $\mathbf{P}$ & Age-deprivation-adj. OR (95\% Cl) & $\mathbf{P}$ \\
\hline White British & $33,540,548$ & $177,855,632$ & Ref & - & Ref & - \\
Asian Other & 466,524 & $3,630,486$ & $0.85(0.85-0.86)$ & $<.001$ & $0.84(0.84-0.84)$ & $<.001(0.87-0.88)$ \\
Bangladeshi & 251,031 & $1,931,539$ & $0.93(0.92-0.93)$ & $<.001$ & $<.001$ \\
Chinese & 140,011 & $1,757,504$ & $0.51(0.51-0.51)$ & $<.001$ & $0.50(0.50-0.50)$ & $<.001$ \\
Indian & 862,039 & $6,116,471$ & $0.88(0.88-0.88)$ & $<.001$ & $0.86(0.85-0.86)$ & $<.001$ \\
Pakistani & 856,301 & $4,705,109$ & $1.28(1.28-1.29)$ & $<.001$ & $1.21(1.21-1.21)$ & $<.001$ \\
Black African & 572,582 & $4,316,123$ & $0.94(0.94-0.95)$ & $<.001$ & $0.90(0.90-0.90)$ & $<.001$ \\
Black Caribbean & 395,784 & $2,559,296$ & $0.90(0.90-0.91)$ & $<.001$ & $0.86(0.86-0.86)$ & $<.001$ \\
Other & 939,127 & $3,466,723$ & $1.83(1.83-1.83)$ & $<.001$ & $1.76(1.76-1.76)$ & $<.001$ \\
White Other & $1,903,020$ & $10,247,030$ & $1.14(1.14-1.14)$ & $<.001$ & $1.13(1.12-1.13)$ & $<.001$ \\
Mixed & 409,131 & $5,555,264$ & $0.55(0.55-0.55)$ & $<.001$ & $0.54(0.53-0.53)$ & $<.001$
\end{tabular}

practice. This raises the question - in general and for further research - whether preventive infrastructures are used optimally by ethnic groups with known higher risk profiles, i.e. whether more could be done to prevent hospitalisation. This particular study was designed to detect ethnic inequalities in admissions overall and further studies would be required to narrow in on particular unmet needs and any specific barriers that ethnic groups may face navigating the healthcare system.

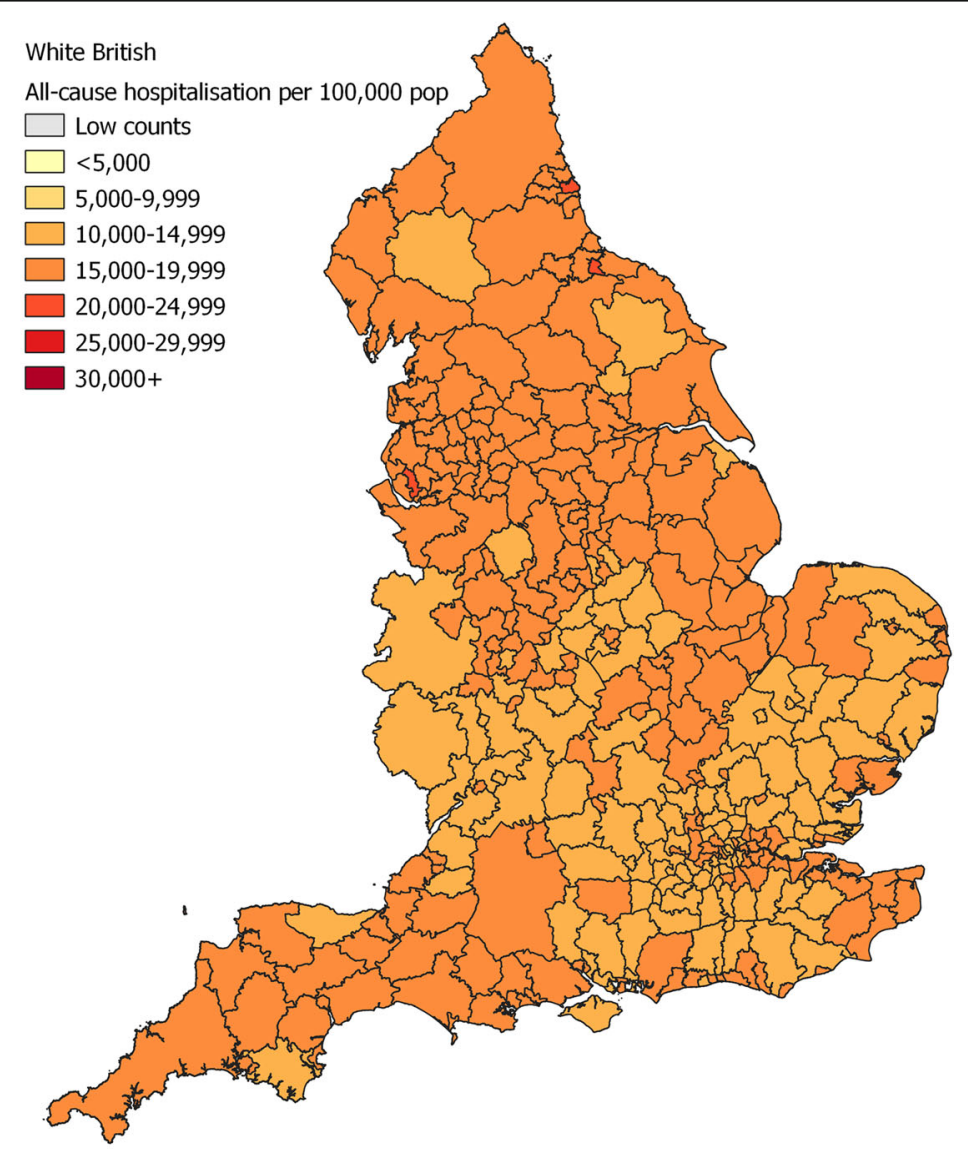

Fig. 1 Map of all-cause hospitalisation per 100,000 population, 2009-2013, for White British 


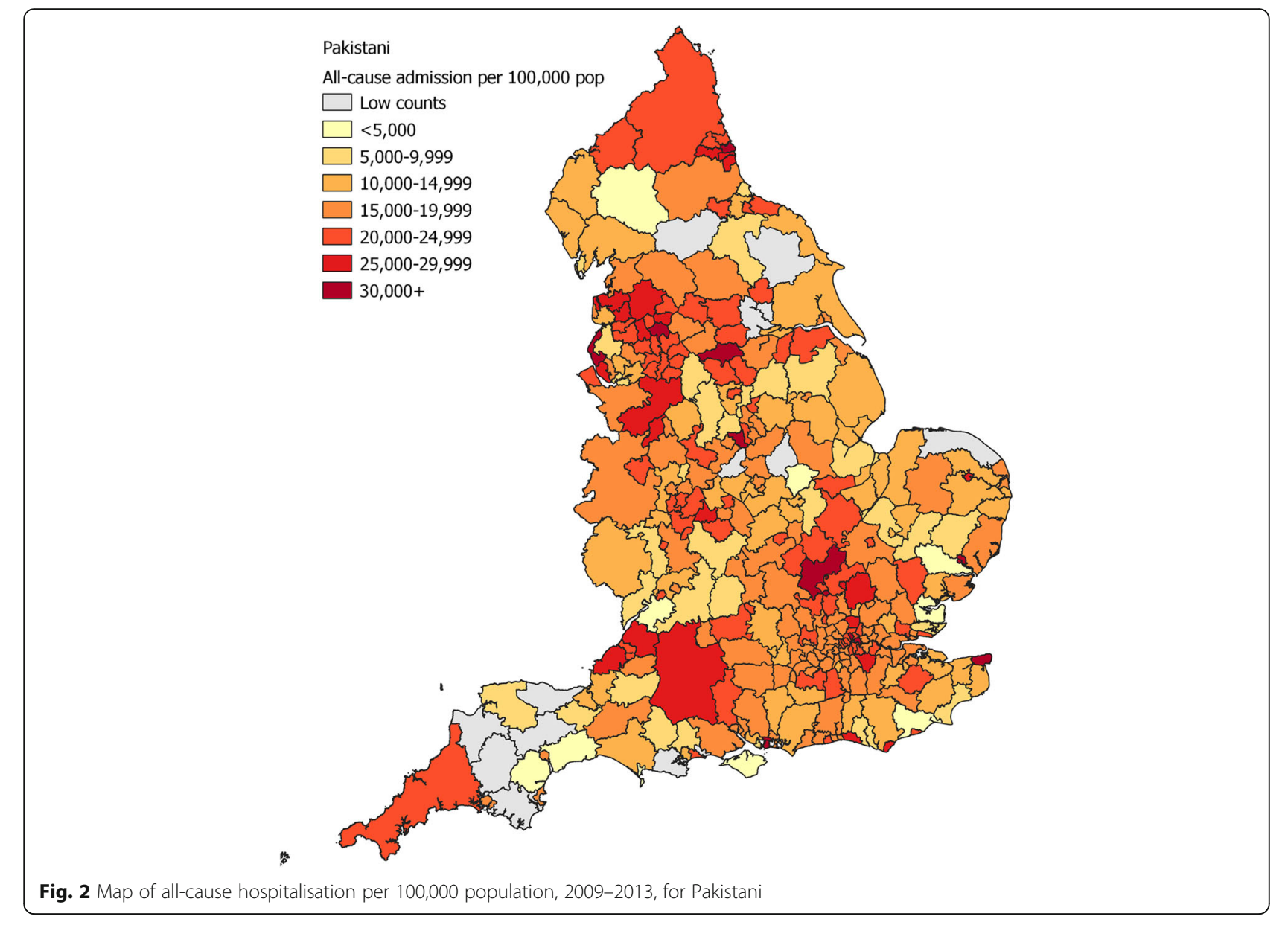

After more than two decades of policies and initiatives to explicitly reduce ethnic inequalities in the UK $[1,2]$, a review published in 2020 disconcertingly found evidence of persistent inequalities across many domains [3]. The review also found ethnic minorities less satisfied with the health services than the White British group and that they reported longer waiting times before being seen by a cancer specialist.

Chinese and mixed ethnic groups typically had much lower admission incidence that the White British group. The Chinese minority are known to have lower hospitalisation risk, good self-reported health [22], and lower mortality risk [32]. While the Chinese minority may have fewer health needs, they might also be less well recorded by NHS services compared to other groups due to a preference for traditional Chinese medicine [33].

Self-reported ethnicity information was missing for $7.3 \%$ of patients and was reduced to $0.2 \%$ by imputing ethnicity from patient surname to give a more complete representation. The sensitivity for the surname-derived information was better in some groups, e.g. more than $90 \%$ for White British and just above $50 \%$ for Black African. These biases must be acknowledged although limited since only $7.3 \%$ of patients in HES had no ethnicity data. The regression analyses in this study however yielded similar results with and without the surname imputation. The differences in sensitivity of the EE software are consistent with other studies and reflect factors such as differences in naming practices, sense of belonging, and migration history [13]. Although the sensitivity of the software is not equal for all groups, the alternatives in most cases are to analyse the data as complete case or imputing as if missing at random. The EE software is currently under development to improve its sensitivity and range not least for more recent expansions of the Census ethnicity classification with groups such as Arab and Roma.

A survey of cancer patients found consistency between NHS-recorded and self-reported ethnicity for $95 \%$ of participants [34]. This is a strength in relation to the use of self-reported ethnicity denominators from the Census. 


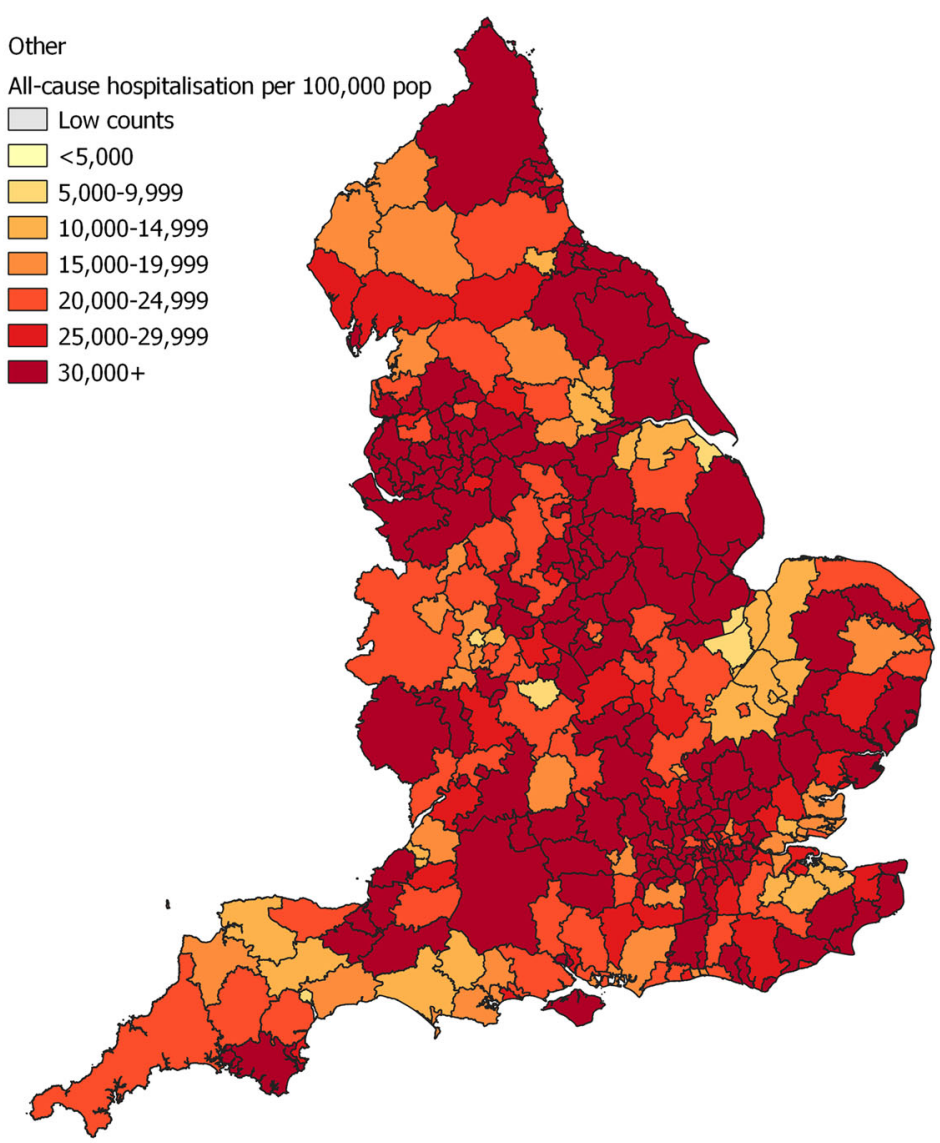

Fig. 3 Map of all-cause hospitalisation per 100,000 population, 2009-2013, for Other ethnic group

\section{Conclusion}

The study found stark disparities between ethnic minorities in hospitalisation for a sweep of conditions. Ethnic inequalities in health are well known for conditions such as cardiovascular diseases, respiratory diseases, and diabetes, but this study also found inequalities in nutritional deficiencies, endocrine disorders, and sense organ diseases.

Further studies would be required to map out the relevant care pathways for ethnic minorities and establish whether preventive measures can be strengthened.

\section{Abbreviations}

UK: United Kingdom; NHS: National Health Service; HES: Hospital Episode Statistics; GBD: Global Burden of Disease; ICD10: 10th version of International Classification of Diseases; EE: Ethnicity Estimator

\section{Supplementary Information}

The online version contains supplementary material available at https://doi. org/10.1186/s12889-021-10923-5.

Additional file 1: Table S1. Age-adjusted odds ratios of admission incidence relative to white majority group. Blue shading shows odds ratios significantly below White British group; red shading above. Darker tones show less than half and more than twice, respectively. Non-significant (ns) results shown on white background. Ethnicity as recorded by NHS.
Table S2. Age- and deprivation-adjusted odds ratios of admission incidence relative to white majority group. Blue shading shows odds ratios significantly below White British group; red shading above. Darker tones show less than half and more than twice, respectively. Non-significant (ns) results shown on white background. Ethnicity as recorded by NHS. Table S3. Age- and deprivation- adjusted odds ratios (OR 95\% Cl) for allcause hospitalisation. Ethnicity as recorded by NHS.

\section{Acknowledgements}

Not applicable.

\section{Authors' contributions}

All authors contributed to conception and design, critically revised the manuscript, gave final approval, and agreed to be accountable for all aspects of work ensuring integrity and accuracy (JK, PAL, JP). JK and PAL contributed to data acquisition. JP contributed to analysis and the first draft of the manuscript.

\section{Funding}

The UK Economic and Social Research Council is acknowledged for its support for the UCL Consumer Data Research Centre (CDRC) enabling this research (Grant ES/L011840/1). The funder had no direct role in relation to the specific study.

\section{Availability of data and materials}

All the data that support the findings of this study are available from NHS Digital subject to ethnical and scientific approval of a study protocol. Researchers wishing to get access to the study data can visit this website: https://digital.nhs.uk/data-and-information/data-tools-and-services/data- 
services/hospital-episode-statistics. The authors of this study had no special access privileges others would not have.

\section{Declarations}

\section{Ethics approval and consent to participate}

Ethical approval was obtained from Bromley REC (Reference: 13/LO/1355). The study was conducted in accordance with relevant guidelines and regulations. As for other non-identifiable database studies, it was not practical nor desirable to obtain consent from each patient. The study only used routinely collected, secondary data and as such involved no experimental components requiring additional protocols and approvals. The HES data licence reference is DARS-NIC-28051-Q3K7L.

\section{Consent for publication}

Not applicable.

\section{Competing interests}

None.

\section{Author details}

${ }^{1}$ Consumer Data Research Centre (CDRC), Department of Geography, University College London (UCL), Gower Street, London WC1E 6BT, UK. ${ }^{2}$ The Bartlett Centre for Advanced Spatial Analysis (CASA), Gower Street, UCL, London WC1E 6BT, UK.

\section{Received: 4 December 2020 Accepted: 23 April 2021}

Published online: 05 May 2021

\section{References}

1. Mackenbach JP. Can we reduce health inequalities? An analysis of the English strategy (1997-2010). J Epidemiol Community Health. 2011;65(7): 568-75. https://doi.org/10.1136/jech.2010.128280.

2. Marmot M, Allen J, Boyce T, Goldblatt P, Morrison J. Health equity in England: the Marmot review 10 years on. The Health Foundation. 2020; https://www.health.org.uk/publications/reports/the-marmot-review-10-yearson. Accessed 10 Jul 2020.

3. Chouhan K, Nazroo J. Health inequalities. In: Ethnicity, race and inequality in the UK - state of the nation. Bristol: Policy Press; 2020. p. 73-92. https://doi. org/10.2307/j.ctv14t47tm.10.

4. Evandrou M, Falkingham J, Feng Z, Vlachantoni A. Ethnic inequalities in limiting health and self-reported health in later life revisited. J Epidemiol Community Health. 2016;70(7):653-62. https://doi.org/10.1136/jech-2015-2 06074.

5. Wang S, Mak H-W. Generational health improvement or decline? Exploring generational differences of British ethnic minorities in six physical health outcomes. Ethn Health. 2018;0:1-14, DOI: https://doi.org/10.1080/13557858.2 018.1557117.

6. Petersen J, Kandt J, Longley PA. Names-based ethnicity enhancement of hospital admissions in England, 1999-2013. Int J Med Inf. 2021;149:104437. https://doi.org/10.1016/j.jmedinf.2021.104437.

7. Kaufman JS, Rushani D, Cooper RS. Nature versus nurture in the explanations for racial/ethnic health disparities: parsing disparities in the era of genome-wide association studies. Oxford: Oxford University Press; 2018. https://oxford.universitypressscholarship.com/view/https://doi.org/10.1093/ oso/9780190465285.001.0001/oso-9780190465285-chapter-7. Accessed 10 Mar 2021

8. $\quad$ Alderwick H, Dixon J. The NHS long term plan. The BMJ. 2019;364:184. https://doi.org/10.1136/bmj.l84

9. Steel N, Ford JA, Newton JN, Davis ACJ, Vos T, Naghavi M, et al. Changes in health in the countries of the UK and 150 English local authority areas 1990-2016: a systematic analysis for the global burden of disease study 2016. Lancet. 2018;392(10158):1647-61. https://doi.org/10.1016/S0140-6736(1 8)32207-4.

10. WHO. ICD-10: International Statistical Classification of Diseases and Related Health Problems 10th Revision. 2016 https://icd.who.int/browse10/2016/en. Accessed 17 Jan 2020.

11. Department for Communities and Local Government, 2015. The English Indices of deprivation 2015. Statistical Release; 2015. p. 1-38.

12. Kandt J, Van Dijk J, Longley PA. Family name origins and inter-generational demographic change in Great Britain. Am Geogr Soc. 2020;110:1726-42.
13. Kandt J, Longley PA. Ethnicity estimation using family naming practices. PLoS One. 2018;13(8):e0201774. https://doi.org/10.1371/journal.pone.0201774.

14. Mateos P, Longley PA, O'Sullivan D. Ethnicity and population structure in personal naming networks. PLoS One. 2011;6(9):e22943. https://doi.org/1 0.1371/journal.pone.0022943.

15. Lakha F, Gorman DR, Mateos P. Name analysis to classify populations by ethnicity in public health: validation of Onomap in Scotland. Public Health. 2011;125(10):688-96. https://doi.org/10.1016/j.puhe.2011. 05.003.

16. Petersen J, Longley P, Gibin M, Mateos P, Atkinson P. Names-based classification of accident and emergency department users. Health Place. 2011;17(5):1162-9. https://doi.org/10.1016/j.healthplace.2010.09.010.

17. Easton S, Pryce G. Not so welcome here? Modelling the impact of ethnic inmovers on the length of stay of home-owners in micro-neighbourhoods. Urban Stud. 2019;56(14):2847-62. https://doi.org/10.1177/0042098018822615.

18. Booth $\mathrm{AL}$, Leigh $\mathrm{A}$, Varganova $\mathrm{E}$. Does ethnic discrimination vary across minority groups? Evidence from a field experiment*. Oxf Bull Econ Stat. 2012;74(4):547-73. https://doi.org/10.1111/j.1468-0084.2011.00664.x.

19. Bernile G, Bhagwat V, Yonker S. Board diversity, firm risk, and corporate policies. J Financ Econ. 2018;127(3):588-612. https://doi.org/10.1016/j. jfineco.2017.12.009.

20. European Commission. Revision of the European Standard Population Report of Eurostat's task force. Luxembourg: Publications Office of the European Union, Methodologies and Working papers; 2013. https://doi. org/10.2785/11470.

21. George J, Mathur R, Shah AD, Pujades-Rodriguez M, Denaxas S, Smeeth L, et al. Ethnicity and the first diagnosis of a wide range of cardiovascular diseases: associations in a linked electronic health record cohort of 1 million patients. PLoS One. 2017;12(6):e0178945. https://doi.org/10.1371/journal. pone.0178945.

22. Health and Social Care Information Centre. Health Survey for England 2004: Health of ethnic minorities 2006. https://digital.nhs.uk/data-andinformation/publications/statistical/health-survey-for-england/health-surveyfor-england-2004-health-of-ethnic-minorities-headline-results.

23. Hippisley-Cox J, Coupland C, Vinogradova Y, Robson J, Minhas R, Sheikh A, et al. Predicting cardiovascular risk in England and Wales: prospective derivation and validation of QRISK2. BMJ. 2008;336(7659):1475-82. https:// doi.org/10.1136/bmj.39609.449676.25.

24. Kristiansen M, Razum O, Tezcan-Güntekin H, Krasnik A. Aging and health among migrants in a European perspective. Public Health Rev. 2016;37(1): 20. https://doi.org/10.1186/s40985-016-0036-1.

25. Sheikh A, Steiner MFC, Cezard G, Bansal N, Fischbacher C, Simpson CR, et al. Ethnic variations in asthma hospital admission, readmission and death: a retrospective, national cohort study of 4.62 million people in Scotland. BMC Med. 2016;14:3.

26. Bhopal RS, Cezard G, Bansal N, Ward HJT, Bhala N. Ethnic variations in five lower gastrointestinal diseases: Scottish health and ethnicity linkage study. BMJ Open. 2014;4(10):e006120. https://doi.org/10.1136/bmjopen-2 014-006120.

27. Bhopal R, Steiner MFC, Cezard G, Bansal N, Fischbacher C, Simpson CR, et al. Risk of respiratory hospitalization and death, readmission and subsequent mortality: Scottish health and ethnicity linkage study. Eur J Pub Health. 2015;25(5):769-74. https://doi.org/10.1093/eurpub/ckv064.

28. Netuveli G, Hurwitz B, Levy M, Fletcher M, Barnes G, Durham SR, et al. Ethnic variations in UK asthma frequency, morbidity, and health-service use: a systematic review and meta-analysis. Lancet. 2005;365(9456):312-7. https:// doi.org/10.1016/S0140-6736(05)17785-X.

29. Goddard AF, James MW, McIntyre AS, Scott BB. Guidelines for the management of iron deficiency anaemia. Gut. 2011;60(10):1309-16. https:// doi.org/10.1136/gut.2010.228874.

30. Kato G, Piel FBJ, Vichinsky E. Sickle cell disease. Nat Rev Dis Primer. 2018;15: 18010.

31. Rauf A, Malik R, Bunce C, Wormald R. The British Asian community eye study: outline of results on the prevalence of eye disease in British Asians with origins from the Indian subcontinent. Indian J Ophthalmol. 2013;61(2): 53-8. https://doi.org/10.4103/0301-4738.107191.

32. Bhopal RS, Gruer L, Cezard G, Douglas A, Steiner MFC, Millard A, et al. Mortality, ethnicity, and country of birth on a national scale, 2001-2013: a retrospective cohort (Scottish health and ethnicity linkage study). PLOS Med. 2018;15(3):e1002515. https://doi.org/10.1371/journal.pmed.1 002515. 
33. Sproston KA, Pitson LB, Walker $E$. The use of primary care services by the Chinese population living in England: examining inequalities. Ethn Health. 2001;6(3-4):189-96. https://doi.org/10.1080/13557850120078116.

34. Saunders CL, Abel GA, El Turabi A, Ahmed F, Lyratzopoulos G. Accuracy of routinely recorded ethnic group information compared with selfreported ethnicity: evidence from the English Cancer patient experience survey. BMJ Open. 2013;3(6):e002882. https://doi.org/10.1136/bmjopen-2 013-002882.

\section{Publisher's Note}

Springer Nature remains neutral with regard to jurisdictional claims in published maps and institutional affiliations.

Ready to submit your research? Choose BMC and benefit from:

- fast, convenient online submission

- thorough peer review by experienced researchers in your field

- rapid publication on acceptance

- support for research data, including large and complex data types

- gold Open Access which fosters wider collaboration and increased citations

- maximum visibility for your research: over $100 \mathrm{M}$ website views per year

At $\mathrm{BMC}$, research is always in progress.

Learn more biomedcentral.com/submissions 